\title{
Heavy water stratification in a low-mass protostar ${ }^{\star}$
}

\author{
A. Coutens ${ }^{1,2}$, C. Vastel $^{1,2}$, S. Cazaux ${ }^{3}$, S. Bottinelli ${ }^{1,2}$, E. Caux ${ }^{1,2}$, C. Ceccarelli ${ }^{4}$, K. Demyk ${ }^{1,2}$, \\ V. Taquet ${ }^{4}$, and V. Wakelam ${ }^{5}$
}

\author{
1 Université de Toulouse, UPS-OMP, IRAP, Toulouse, France \\ e-mail: acoutens@nbi.dk, cvastel@irap.omp.eu \\ 2 CNRS, Institut de Recherche en Astrophysique et Planétologie, 9 Av. Colonel Roche, BP 44346, 31028 Toulouse Cedex 4, France \\ 3 Kapteyn Astronomical Institute, PO Box 800, 9700AV Groningen, The Netherlands \\ ${ }^{4}$ Institut de Planétologie et d'Astrophysique de Grenoble, UMR 5274, UJF-Grenoble 1/CNRS, 38041 Grenoble, France \\ 5 CNRS and Université de Bordeaux, Observatoire Aquitain des Sciences de l'Univers, 2 rue de l'Observatoire, BP 89, 33271 Floirac, \\ France
}

Received 20 December 2012 / Accepted 18 March 2013

\section{ABSTRACT}

\begin{abstract}
Context. Despite the low elemental deuterium abundance in the Galaxy, enhanced molecular deuterium fractionation has been found in the environments of low-mass star-forming regions and, in particular, the Class 0 protostar IRAS 16293-2422.

Aims. The key program Chemical HErschel Surveys of Star forming regions (CHESS) aims at studying the molecular complexity of the interstellar medium. The high sensitivity and spectral resolution of the Herschel/HIFI (Heterodyne Instrument for Far-Infrared) instrument provide a unique opportunity to observe the fundamental $1_{1,1}-0_{0,0}$ transition of ortho- $\mathrm{D}_{2} \mathrm{O}$ at $607 \mathrm{GHz}$ and the higher energy $2_{1,2}-1_{0,1}$ transition of para- $\mathrm{D}_{2} \mathrm{O}$ at $898 \mathrm{GHz}$, both of which are inaccessible from the ground.

Methods. The ortho- $\mathrm{D}_{2} \mathrm{O}$ transition at $607 \mathrm{GHz}$ was previously detected. We present in this paper the first tentative detection for the para- $\mathrm{D}_{2} \mathrm{O}$ transition at $898 \mathrm{GHz}$. The spherical Monte Carlo radiative transfer code RATRAN was used to reproduce the observed line profiles of $\mathrm{D}_{2} \mathrm{O}$ with the same method that was used to reproduce the $\mathrm{HDO}$ and $\mathrm{H}_{2}{ }^{18} \mathrm{O}$ line profiles in IRAS 16293-2422.

Results. As for HDO, the absorption component seen on the $\mathrm{D}_{2} \mathrm{O}$ lines can only be reproduced by adding an external absorbing layer, possibly created by the photodesorption of the ices at the edges of the molecular cloud. The $\mathrm{D}_{2} \mathrm{O}$ column density is found to be about $2.5 \times 10^{12} \mathrm{~cm}^{-2}$ in this added layer, leading to a $\mathrm{D}_{2} \mathrm{O} / \mathrm{H}_{2} \mathrm{O}$ ratio of about $0.5 \%$. At a $3 \sigma$ uncertainty, upper limits of $0.03 \%$ and $0.2 \%$ are obtained for this ratio in the hot corino and the colder envelope of IRAS 16293-2422, respectively.

Conclusions. The deuterium fractionation derived in our study suggests that the ices present in IRAS 16293-2422 formed on warm dust grains $(\sim 15-20 \mathrm{~K})$ in dense $\left(\sim 10^{4}-5 \times 10^{4} \mathrm{~cm}^{-3}\right)$ translucent clouds. These results allow us to address the earliest phases of star formation and the conditions in which ices form.
\end{abstract}

Key words. astrochemistry - ISM: individual objects: IRAS 16293-2422 - ISM: molecules

\section{Introduction}

Despite the low elemental deuterium abundance in the local in-

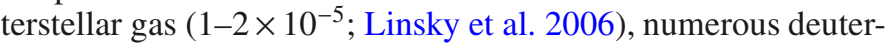
ated molecules have been detected with enhanced $\mathrm{D} / \mathrm{H}$ ratios in the environments of low-mass star-forming regions. The doubly deuterated form of water, $\mathrm{D}_{2} \mathrm{O}$, has been in particular detected in the solar-type protostar IRAS 16293-2422 (hereafter IRAS 16293), which is well known for its high deuterium fractionation: the fundamental $1_{1,0}-1_{0,1}$ para- $\mathrm{D}_{2} \mathrm{O}$ transition at $317 \mathrm{GHz}$ was detected by Butner et al. (2007) using the James Clerk Maxwell Telescope (JCMT), whereas the fundamental $1_{1,1}-0_{0,0}$ ortho- $\mathrm{D}_{2} \mathrm{O}$ transition at $607 \mathrm{GHz}$ was discovered with the Heterodyne Instrument for Far-Infrared (HIFI) instrument onboard the Herschel Space Observatory by Vastel et al. (2010). The observed line profile at $317 \mathrm{GHz}$ shows a component in emission in addition to a deep and narrow absorption. The emission component has been attributed to heavy water in the hot corino of this source, where the grain ices are

* Based on Herschel/HIFI observations. Herschel is an ESA space observatory with scientific instruments provided by European-led principal Investigator consortia and with important participation from NASA. sublimated and released into the gas phase (Ceccarelli et al. 2000; Bottinelli et al. 2004). The absorption component, whose linewidth is $0.5 \mathrm{~km} \mathrm{~s}^{-1}$, is seen in both transitions at the velocity of $4.2 \mathrm{~km} \mathrm{~s}^{-1}$. It probably originates in the foreground gas (molecular cloud and cold envelope), which is studied through the fundamental HDO transition at 465 and $894 \mathrm{GHz}$ (Coutens et al. 2012). Both transitions present deep absorption features at the same velocity as the $\mathrm{D}_{2} \mathrm{O}$ component. The OD molecule, a key species in the formation of deuterated water on grains, also shows a deep absorbing component (Parise et al. 2012) and most certainly arises from the same region as water and its deuterated forms. Besides, numerous absorption lines of several nitrogen molecules $\left(\mathrm{NH}, \mathrm{ND}, \mathrm{NH}_{2}\right.$, and $\mathrm{NH}_{3}$ ) are detected towards the IRAS 16293 source (Bacmann et al. 2010; Hily-Blant et al. 2010), suggesting an origin of these species in a cold environment. Coutens et al. (2012) used the spherical Monte Carlo radiative transfer code RATRAN (Hogerheijde \& van der Tak 2000) to reproduce the line profiles of the two HDO fundamental transitions as well as eleven other detected lines by assuming an abundance jump at $100 \mathrm{~K}$ (Fraser et al. 2001). From the emission line profiles, the derived HDO inner abundance $(T \geq 100 \mathrm{~K})$ is about $1.7 \times 10^{-7}$, whereas the HDO outer abundance $(T<100 \mathrm{~K})$ is estimated at about $8 \times 10^{-11}$. A water-rich 
absorbing layer is, however, required in front of the envelope to reproduce the HDO absorption components observed at 465 and $894 \mathrm{GHz}$. This layer may result from the photodesorption of the ices at the edges of the molecular cloud, as predicted by chemical models (e.g., Hollenbach et al. 2009).

A non-LTE (local thermal equilibrium) modeling has been performed by Vastel et al. (2010) to estimate the ortho-to-para $\mathrm{D}_{2} \mathrm{O}$ ratio in the cold $(<30 \mathrm{~K})$ dense $\left(<5 \times 10^{6} \mathrm{~cm}^{-3}\right)$ cloud surrounding the IRAS 16293 protostar, adopting the density and temperature profiles of the envelope (Crimier et al. 2010). Vastel et al. (2010) considered the first two levels of each $\mathrm{D}_{2} \mathrm{O}$ form and used the computed collisional rates for the two fundamental deexcitation transitions of ortho- and para- $\mathrm{D}_{2} \mathrm{O}$ with para- $\mathrm{H}_{2}$ in the 10-30 K range (Scribano et al. 2010). Their computation yields an ortho-to-para ratio of $1.1 \pm 0.4$ at a $1 \sigma$ level of uncertainty with the corresponding column densities $N$ (ortho) $=$ $(8.7 \pm 2.1) \times 10^{11} \mathrm{~cm}^{-2}$ and $N($ para $)=(7.8 \pm 2.6) \times 10^{11} \mathrm{~cm}^{-2}$. This ratio is lower than 2.6 at a $3 \sigma$ level of uncertainty, taking into account the statistical error as well as the overall calibration budget for the HIFI band $1 \mathrm{~b}$. The comparison between the upper value of the measured $\mathrm{D}_{2} \mathrm{O}$ ortho-to-para ratio and the thermal equilibrium value shows that they are consistent with a gas at a temperature higher than about $15 \mathrm{~K}$ and, therefore, with the assumed absorbing gas location.

The present paper aims at determining the $\mathrm{D}_{2} \mathrm{O}$ abundance distribution throughout the whole protostellar envelope of IRAS 16293, from the hot corino to the foreground water-rich absorbing layer (see Fig. 2 in Coutens et al. 2012). Using the previous results obtained in a similar way by Coutens et al. (2012) for $\mathrm{HDO}$ and $\mathrm{H}_{2} \mathrm{O}$, the derived $\mathrm{D}_{2} \mathrm{O} / \mathrm{H}_{2} \mathrm{O}$ and $\mathrm{HDO} / \mathrm{H}_{2} \mathrm{O}$ ratios are then discussed and compared with a grain-surface chemical model (Cazaux et al. 2011). The paper is organized as follows. First, we describe the observations as well as their reduction in Sect. 2. Then we present the modeling and the results in Sect. 3. Finally, we discuss the results in Sect. 4 and conclude in Sect. 5.

\section{Observations}

In the framework of the Chemical HErschel Surveys of Star forming regions (CHESS) key program (Ceccarelli et al. 2010), we observed the solar-type protostar IRAS 16293 with the Herschel/HIFI instrument (Pilbratt et al. 2010; de Graauw et al. 2010). The targeted coordinates were $\alpha_{2000}=16^{\mathrm{h}} 32^{\mathrm{m}} 22^{\mathrm{s}} .75$, $\delta_{2000}=-24^{\circ} 28^{\prime} 34.2^{\prime \prime}$, a position at equal distance from the binary components A and B. A full spectral coverage of bands $1 \mathrm{~b}$ and $3 \mathrm{~b}$ was performed on 2010 March 2 and 2010 March 19 respectively, using the HIFI spectral scan double beam switch (DBS) mode with optimization of the continuum. The fundamental ortho- $\mathrm{D}_{2} \mathrm{O} 1_{1,1}-0_{0,0}$ transition lies in this frequency coverage at $607.349 \mathrm{GHz}$ whereas the para- $\mathrm{D}_{2} \mathrm{O} 2_{1,2}-1_{1,0}$ transition lies at $897.947 \mathrm{GHz}$. The HIFI Wide Band Spectrometer (WBS) was used, providing a spectral resolution of $1.1 \mathrm{MHz}$ $\left(\sim 0.55 \mathrm{~km} \mathrm{~s}^{-1}\right.$ at $600 \mathrm{GHz}$ and $\sim 0.37 \mathrm{~km} \mathrm{~s}^{-1}$ at $\left.900 \mathrm{GHz}\right)$ over an instantaneous bandwidth of $4 \times 1 \mathrm{GHz}$. The ortho$\mathrm{D}_{2} \mathrm{O} 1_{1,1}-0_{0,0}$ transition was re-observed in February 2011 with the High Resolution Spectrometer (HRS) to benefit from a higher spectral resolution $\left(\sim 0.06 \mathrm{~km} \mathrm{~s}^{-1}\right)$. We consequently use these new observations hereafter. At $610 \mathrm{GHz}(900 \mathrm{GHz})$, the beam size is about $35^{\prime \prime}\left(24^{\prime \prime}\right.$ resp.) and the measured main beam efficiency is 0.75 ( 0.74 resp.), whereas the forward efficiency is 0.96 in both cases (Roelfsema et al. 2012). The DBS reference positions were situated approximately $3^{\prime}$ east and west of the source.
The data reduction of the para- $\mathrm{D}_{2} \mathrm{O} 2_{1,2}-1_{1,0}$ transition at $898 \mathrm{GHz}$ was performed similarly to the $\mathrm{HDO}$ and $\mathrm{H}_{2}{ }^{18} \mathrm{O}$ lines studied by Coutens et al. (2012). This transition was observed eight times (four in lower sideband and four in upper sideband) for each polarization in the selected observing mode. To produce the final spectra, the observations that were processed using the standard HIFI pipeline up to level 2 with the ESAsupported package Herschel interactive processing environment (HIPE) 5.1 (Ott 2010) were exported to the GILDAS/CLASS ${ }^{1}$ software. We then baseline subtracted and averaged the $\mathrm{H}$ and $\mathrm{V}$ polarizations at the line frequency, weighting them by the observed noise for each spectra. We verified for all spectra that no emission from other species was present in the image band. This transition had not been discovered when Vastel et al. (2010) published their results with an early version (3.01) of the HIPE software, using the sideband deconvolution method on all bands. The present data reduction, which uses the selected spectra realigned on one single transition and averaged after an accurate baseline subtraction and rms weighting, leads to a lower noise level than the one obtained with the standard HIPE deconvolution routine, and allows us to present the first tentative detection of a high-energy level transition of deuterated water (see Fig. 2). For the pointed HRS data at $607 \mathrm{GHz}$, the observations of the $\mathrm{H}$ and $\mathrm{V}$ polarizations were processed with the ESA-supported package HIPE 8.0 (Ott 2010) up to level 2 and then exported to the GILDAS/CLASS software for the baselines to be subtracted and the two polarizations averaged. HIFI operates as a double sideband (DSB) receiver. From the in-orbit performances of the instrument (Roelfsema et al. 2012), a sideband ratio of unity is assumed for the observed $\mathrm{D}_{2} \mathrm{O}$ transitions seen in absorption against the continuum (bands $1 \mathrm{~b}$ and $3 \mathrm{~b}$ ). The continuum values observed over the frequency range of the whole bands are closely fitted by straight lines. The continuum derived from the polynomial fit at the considered frequency and divided by two to obtain the single sideband (SSB) continuum was added to the spectra.

The parameters of the lines, taking into account the orthoand para- $\mathrm{D}_{2} \mathrm{O}$ forms (within the CASSIS software ${ }^{2}$ ) separated from the Cologne Database for Molecular Spectroscopy (Müller et al. 2005; Brünken et al. 2007), are reported in Table 1. The fundamental ortho- $\mathrm{D}_{2} \mathrm{O}$ transition at $607.349 \mathrm{GHz}$ is clearly detected in absorption against the strong continuum $(\sim 0.3 \mathrm{~K})$. We present here the first tentative detection of the para- $\mathrm{D}_{2} \mathrm{O} 2_{1,2}-1_{0,1}$ transition at $897.95 \mathrm{GHz}$, seemingly in absorption against the continuum $(\sim 0.75 \mathrm{~K})$. In the same table, we also report the parameters of the para- $\mathrm{D}_{2} \mathrm{O} 1_{1,0}-1_{0,1}$ fundamental line previously observed at $316.800 \mathrm{GHz}$ with the JCMT by Butner et al. (2007). We used the line decomposition in three Gaussian components derived by Vastel et al. (2010): two of them are attributed to the broad emission component and the deep absorbing line of $\mathrm{D}_{2} \mathrm{O}$, whereas an additional component emitted at $10.1 \mathrm{~km} \mathrm{~s}^{-1}$ is likely due to the $7_{0}-6_{0}+\mathrm{CH}_{3} \mathrm{OD}$ line at $316.795 \mathrm{GHz}$. This latter component was subtracted in Fig. 2. The $2_{0,2}-1_{1,1}$ ortho transition at $468.25 \mathrm{GHz}$ (see Fig. 1 ) has not been observed, and we consequently only provide a prediction with the modeling.

Observations were recently carried out with the Atacama Pathfinder EXperiment (APEX) telescope between 270 and $330 \mathrm{GHz}$ to complete The IRAS16293-2422 Millimeter And Submillimeter Spectral Survey (TIMASSS; Caux et al. 2011). While the para- $\mathrm{D}_{2} \mathrm{O}$ fundamental $1_{1,0}-1_{0,1}$ line at $317 \mathrm{GHz}$

\footnotetext{
1 http://wWw . iram. fr/IRAMFR/GILDAS

2 Developed by IRAP-UPS/CNRS: http://cassis .irap.omp.eu
} 
Table 1. Derived parameters of the absorbing components of the ortho- and para- $\mathrm{D}_{2} \mathrm{O}$ fundamental lines.

\begin{tabular}{lcccccccccc}
\hline \hline Species & Transition & $\begin{array}{c}\text { Frequency } \\
(\mathrm{GHz})\end{array}$ & $\begin{array}{c}A_{\mathrm{ij}} \\
\left(\mathrm{s}^{-1}\right)\end{array}$ & $\begin{array}{c}E_{\mathrm{up}} \\
(\mathrm{K})\end{array}$ & Telescope & $\begin{array}{c}\int T_{\mathrm{abs}} \mathrm{d} v \\
\left(\mathrm{mK} \mathrm{km} \mathrm{s}^{-1}\right)\end{array}$ & $\begin{array}{c}T_{\mathrm{abs}} \\
(\mathrm{mK})\end{array}$ & $\begin{array}{c}F W H M \\
\left(\mathrm{~km} \mathrm{~s}^{-1}\right)\end{array}$ & $\begin{array}{c}V_{L S R} \\
\left(\mathrm{~km} \mathrm{~s}^{-1}\right)\end{array}$ & $\begin{array}{c}\mid T_{\mathrm{abs}} / / T_{\mathrm{C}} \\
\text { (n) }\end{array}$ \\
\hline ortho- $\mathrm{D}_{2} \mathrm{O}$ & $1_{1,1}-0_{0,0}$ & 607.34945 & $2.95 \times 10^{-3}$ & 29.1 & HIFI 1b/HRS & $-90 \pm 8$ & $-199 \pm 8$ & $0.43 \pm 0.02$ & $4.22 \pm 0.01$ & $0.64 \pm 0.03$ \\
para- $\mathrm{D}_{2} \mathrm{O}$ & $1_{1,0}-1_{0,1}$ & 316.79981 & $6.29 \times 10^{-4}$ & 32.6 & JCMT & $-120 \pm 49$ & $-220 \pm 30$ & $0.55 \pm 0.15$ & $4.15 \pm 0.04$ & $0.57 \pm 0.08$ \\
para- $\mathrm{D}_{2} \mathrm{O}$ & $2_{1,2}-1_{0,1}$ & 897.94711 & $8.61 \times 10^{-3}$ & 60.5 & HIFI 3b/WBS & $-73 \pm 13$ & $-167 \pm 12$ & $0.41 \pm 0.04$ & $4.04 \pm 0.02$ & $0.22 \pm 0.02$ \\
\hline
\end{tabular}
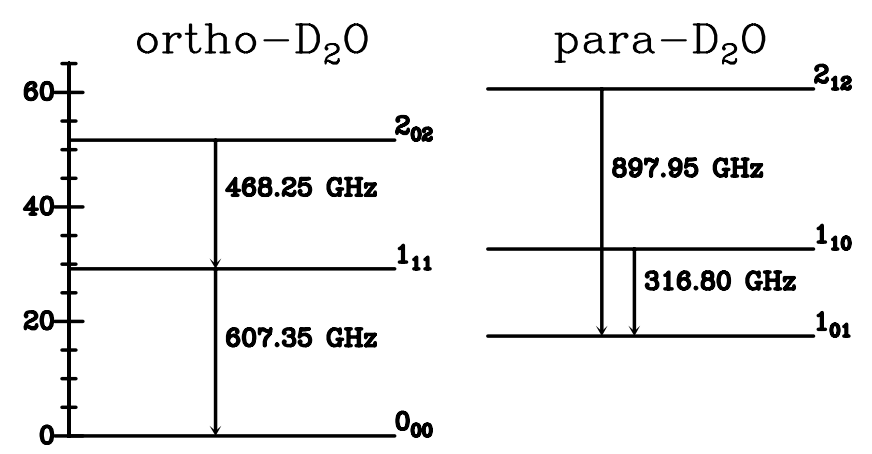

Fig. 1. Energy levels for the lowest rotational transitions of ortho- and para- $\mathrm{D}_{2} \mathrm{O}$.

is also detected in this survey, it shows a much lower signalto-noise ratio than the one observed with JCMT (Butner et al. 2007). This observation is nevertheless useful due to the accurate continuum at this frequency $\left(0.235 \mathrm{~K}\right.$ in $\left.T_{A}^{*}\right)$. The beam efficiency is 0.73 and the forward efficiency 0.97 .

\section{Modeling}

In order to determine the $\mathrm{D}_{2} \mathrm{O} / \mathrm{HDO}$ and $\mathrm{D}_{2} \mathrm{O} / \mathrm{H}_{2} \mathrm{O}$ ratios within the different components of this source (hot corino, colder envelope, and external absorption layer), we developed the same modeling as performed in Coutens et al. (2012) for the HDO and $\mathrm{H}_{2}{ }^{18} \mathrm{O}$ species with the RATRAN radiative transfer code (Hogerheijde \& van der Tak 2000). We employed the orthoand para- $\mathrm{D}_{2} \mathrm{O}$ collisional coefficients calculated with ortho- and para- $\mathrm{H}_{2}$ by Faure et al. (2012) in the $5-100 \mathrm{~K}$ range. We assumed that the ortho-to-para $\mathrm{D}_{2} \mathrm{O}$ ratio is $2: 1$, compatible with the results from Vastel et al. (2010). Using the same parameters for the modeling (see details in Coutens et al. 2012), we realized that the continuum modeled at $317 \mathrm{GHz}(0.38 \mathrm{~K})$ is not consistent with the continuum observed by Butner et al. (2007) at $0.85 \mathrm{~K}$. To determine whether this divergence comes from the modeling or from the observations, we used the recent APEX observations. The SSB continuum observed with APEX is $0.31 \mathrm{~K}\left(T_{\mathrm{mb}}\right)$. Our modeling predicts a $0.29 \mathrm{~K}$ continuum value at the same frequency. From the comparison between transitions from overlapping frequency ranges, we can estimate the calibration to be $20 \%$. The value estimated from the modeling is therefore consistent with the APEX continuum value, and in the following we will use the $0.38 \mathrm{~K}$ predicted by our modeling for the continuum of the $317 \mathrm{GHz}$ transition observed with JCMT.

With the source structure (hot corino + colder envelope) derived by Crimier et al. (2010), the modeling is not able to reproduce any absorption feature for the $\mathrm{D}_{2} \mathrm{O}$ fundamental transitions. The overall absorption can only be reproduced by the external absorbing layer, which was discovered by Coutens et al. (2012) in their efforts to reproduce the absorption components seen on the HDO fundamental transitions. Assuming an $\mathrm{H}_{2}$ density of $\sim 10^{3}-10^{4} \mathrm{~cm}^{-3}$, a kinetic temperature of $\sim 10-30 \mathrm{~K}$ and a b-Doppler parameter of $0.3 \mathrm{~km} \mathrm{~s}^{-1}$ (turbulence), the ortho$\mathrm{D}_{2} \mathrm{O}$ (respectively para- $\mathrm{D}_{2} \mathrm{O}$ ) column density in this layer is about $1.4 \times 10^{12} \mathrm{~cm}^{-2}$ (respectively $1.1 \times 10^{12} \mathrm{~cm}^{-2}$ ). Our modeling predicts that the $2_{1,2}-1_{0,1}$ para- $\mathrm{D}_{2} \mathrm{O}$ transition is expected in absorption as well, tracing the external absorbing layer. The tentatively detected absorbing component at $898 \mathrm{GHz}$ is consequently in perfect agreement with the modeling (see Fig. 2). The kinetic temperature $(\sim 10-30 \mathrm{~K})$ has a limited influence on the absorbing transitions, but hydrogen density seems to have more influence. With a $10^{5} \mathrm{~cm}^{-3}$ density, the absorption depth is less pronounced for the fundamental $1_{1,0}-1_{0,1}$ and $1_{1,1}-0_{0,0}$ transitions at 317 and $607 \mathrm{GHz}$, requiring a higher $\mathrm{D}_{2} \mathrm{O}$ column density. However, the higher energy para- $\mathrm{D}_{2} \mathrm{O}$ transition absorption is overestimated compared to the observations, suggesting a lower $\mathrm{H}_{2}$ density. From the above estimations, the $\mathrm{D}_{2} \mathrm{O}$ orthoto-para ratio is, in the absorbing layer, about 1.3 , close to the value determined by Vastel et al. (2010). However, taking into account the observation and modeling uncertainties, we cannot exclude that the measured value might be consistent with the $2: 1$ value at equilibrium. The quoted column densities are slightly higher (less than a factor 2) than those estimated by Vastel et al. (2010), but within their $3 \sigma$ uncertainties. If this absorbing layer is produced by photodesorption at $A_{V} \sim 1-4$ mag as predicted by Hollenbach et al. (2009), the $\mathrm{D}_{2} \mathrm{O}$ abundance in this layer should be about $6.6 \times 10^{-10}-2.7 \times 10^{-9}$, using the relation between the $\mathrm{H}_{2}$ column density and the visual extinction, $N\left(\mathrm{H}_{2}\right) / A_{V}=9.4 \times 10^{20} \mathrm{~cm}^{-2} \mathrm{mag}^{-1}$ (Frerking et al. 1982).

To estimate the $\mathrm{D}_{2} \mathrm{O}$ abundances in the hot corino $\left(X_{\text {in }}\right)$ and the outer envelope $\left(X_{\text {out }}\right)$ of IRAS 16293, we ran a grid of models that take into account the structure of Crimier et al. (2010) as well as the foregound absorbing layer previously defined. The best-fit modeling is then obtained for an internal abundance $X_{\text {in }}=7 \times 10^{-10}$ and an external abundance $X_{\text {out }}=5 \times 10^{-12}$ from the $\chi^{2}$ minimization (see Fig. 3) computed on the line profiles according to the following formalism:

$\chi^{2}=\sum_{i=1}^{N} \sum_{j=1}^{n_{\text {chan }}} \frac{\left(T_{\text {obs }, i j}-T_{\text {mod }, i j}\right)^{2}}{\left(r m s_{i}\right)^{2}}$

with $N$ the number of lines $i, n_{\text {chan }}$ the number of channels $j$ for each line, $T_{\mathrm{obs}, i j}$ and $T_{\mathrm{mod}, i j}$ the intensity observed and predicted by the RATRAN non-LTE modeling respectively in the channel $j$ of the line $i$ and $r m s_{\mathrm{i}}$ the rms of the line $i$. Here, the reduced $\chi^{2}$ is equal to 2.5 . At $2 \sigma$, the inner and outer abundances ranges are $X_{\text {in }}=\left[9 \times 10^{-11}-1.1 \times 10^{-9}\right]$ and $X_{\text {out }}=$ $\left[8 \times 10^{-13}-1.1 \times 10^{-11}\right]$. Only upper limits can, however, be given at $3 \sigma$, with $X_{\text {in }} \leq 1.3 \times 10^{-9}$ and $X_{\text {out }} \leq 1.3 \times 10^{-11}$. The best-fit modeling is shown in Fig. 2.

To check the influence on the ortho-to-para ratio assumed for $\mathrm{D}_{2} \mathrm{O}$ in the protostellar envelope (i.e., the equilibrium value of 2:1), we ran another grid of models with an ortho-to-para $\mathrm{D}_{2} \mathrm{O}$ ratio equal to 1.3 in the envelope, i.e., the same as estimated in the absorbing layer. The results in both cases are quite in agreement and do not allow us to conclude with an ortho-to-para ratio different from the equilibrium value $2: 1$. In addition, we checked that the para- $\mathrm{D}_{2} \mathrm{O}$ emission line predicted at $317 \mathrm{GHz}$ 
Table 2. $\mathrm{HDO} / \mathrm{H}_{2} \mathrm{O}, \mathrm{D}_{2} \mathrm{O} / \mathrm{HDO}$, and $\mathrm{D}_{2} \mathrm{O} / \mathrm{H}_{2} \mathrm{O}$ abundance ratios estimated towards the protostar IRAS 16293 .

\begin{tabular}{l|c|c|c|c|c}
\hline \hline \multirow{2}{*}{} & \multicolumn{2}{|c|}{ Hot corino } & \multicolumn{2}{c|}{ Outer envelope } & Photodesorption layer \\
\cline { 2 - 6 } & Best-fit & $3 \sigma$ & Best-fit & $3 \sigma$ & $A_{V} \sim 1-4$ mag \\
\hline $\mathrm{HDO}^{a}$ & $1.8 \times 10^{-7}$ & $1.4-2.4 \times 10^{-7}$ & $8 \times 10^{-11}$ & $5.5-10.6 \times 10^{-11}$ & $\sim 0.6-2.4 \times 10^{-8}$ \\
$\mathrm{H}_{2} \mathrm{O}^{a, b}$ & $1 \times 10^{-5}$ & $4.7-40.0 \times 10^{-6}$ & $1.5 \times 10^{-8}$ & $7.0-22.5 \times 10^{-9}$ & $\sim 1.3-5.3 \times 10^{-7}$ \\
$\mathrm{D}_{2} \mathrm{O}$ & $7 \times 10^{-10}$ & $\leq 1.3 \times 10^{-9}$ & $5 \times 10^{-12}$ & $\leq 1.3 \times 10^{-11}$ & $\sim 6.6-27 \times 10^{-10}$ \\
$\mathrm{HDO} / \mathrm{H}_{2} \mathrm{O}$ & $1.8 \%$ & $0.4 \%-5.1 \%$ & $0.5 \%$ & $0.3 \%-1.5 \%$ & $\sim 4.8 \%^{c}$ \\
$\mathrm{D}_{2} \mathrm{O} / \mathrm{HDO}$ & $0.4 \%$ & $\leq 0.9 \%$ & $6.3 \%$ & $\leq 23 \%$ & $\sim 10.8 \%$ \\
$\mathrm{D}_{2} \mathrm{O} / \mathrm{H}_{2} \mathrm{O}$ & $0.007 \%$ & $\leq 0.03 \%$ & $0.03 \%$ & $\leq 0.2 \%$ & $\sim 0.5 \% c$ \\
\hline
\end{tabular}

Notes. ${ }^{(a)}$ The $\mathrm{HDO}$ and $\mathrm{H}_{2} \mathrm{O}$ abundances from Coutens et al. (2012) were recalculated with the $\chi^{2}$ minimization shown in Eq. (1). ${ }^{(b)}$ The $\mathrm{H}_{2} \mathrm{O}$ abundances are obtained from the $\mathrm{H}_{2}^{18} \mathrm{O}$ data using the collision rates with $\mathrm{H}_{2}$ calculated by Daniel et al. (2011). The $\mathrm{H}_{2}^{16} \mathrm{O} / \mathrm{H}_{2}^{18} \mathrm{O}$ ratio is assumed to be 500. ${ }^{(c)}$ The ratios remain valid if the absorbing layer is not due to the photodesorption.

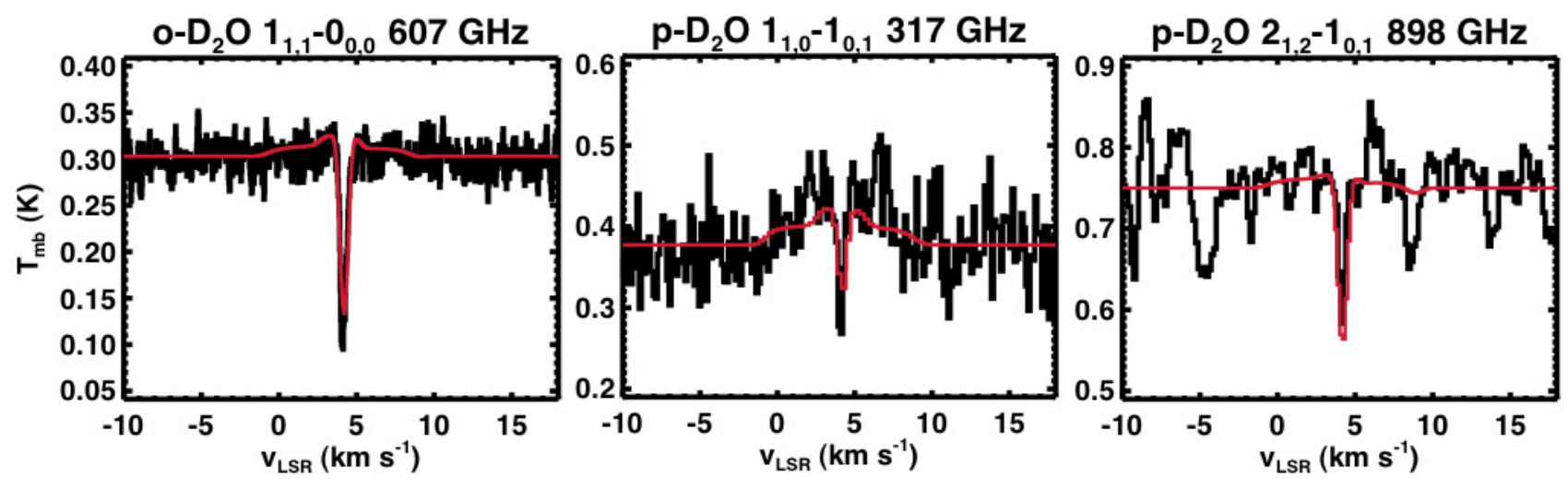

Fig. 2. In black: $\mathrm{D}_{2} \mathrm{O}$ transitions observed with $\mathrm{HIFI}$ and JCMT. The $\mathrm{CH}_{3} \mathrm{OD}\left(7_{0}-6_{0}+\right)$ transition at $316.795074 \mathrm{GHz}$ contaminating the para- $\mathrm{D}_{2} \mathrm{O}\left(1_{1,0}-1_{0,1}\right)$ line profile has been subtracted for a direct comparison with the $\mathrm{D}_{2} \mathrm{O}$ best-fit model. In red: best-fit model obtained when adding an absorbing layer with $N\left(\right.$ para $\left.-\mathrm{D}_{2} \mathrm{O}\right)=1.1 \times 10^{12} \mathrm{~cm}^{-2}$ and $N\left(\right.$ ortho- $\left.-\mathrm{D}_{2} \mathrm{O}\right)=1.4 \times 10^{12} \mathrm{~cm}^{-2}$. The resulting inner abundance is $7 \times 10^{-10}$ and the outer abundance is $5 \times 10^{-12}$.

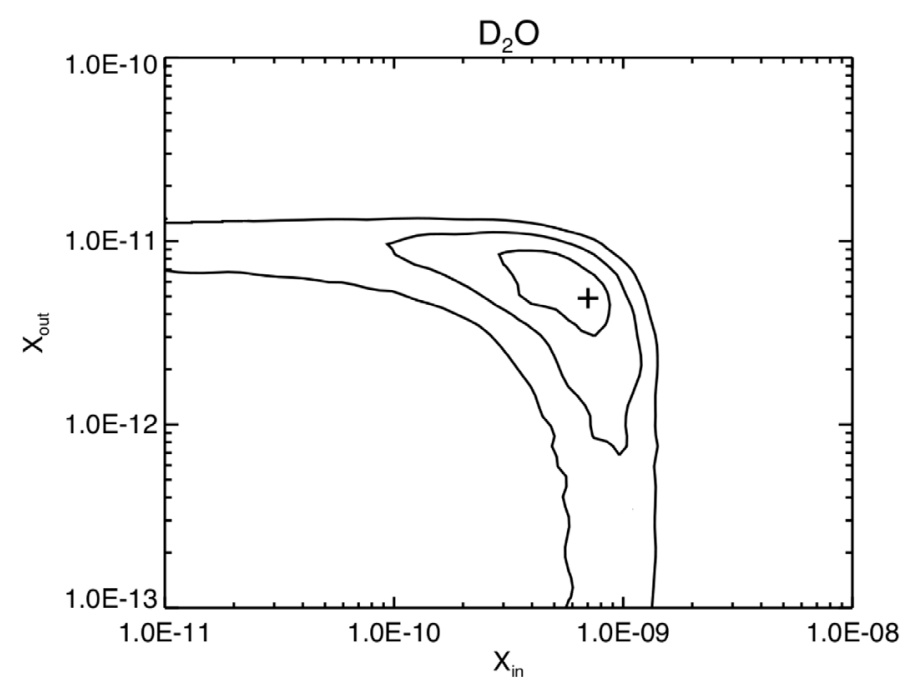

Fig. 3. $\chi^{2}$ contours at $1 \sigma, 2 \sigma$, and $3 \sigma$ obtained when adding an absorption layer with a para- $\mathrm{D}_{2} \mathrm{O}$ column density of $1.1 \times 10^{12} \mathrm{~cm}^{-2}$ and ortho- $\mathrm{D}_{2} \mathrm{O}$ column density of $1.4 \times 10^{12} \mathrm{~cm}^{-2}$. The best-fit model is represented by the symbol "+".

was consistent with the line observed with APEX. Regarding the $2_{0,2}-1_{1,1}$ ortho transition at $468 \mathrm{GHz}$, we predict that the line only shows an emission component with an intensity about $0.1 \mathrm{~K}$ $\left(T_{\mathrm{mb}}\right)$ if observed with APEX.

\section{Discussion}

\subsection{Ortho-to-para $\mathrm{D}_{2} \mathrm{O}$ ratio}

At the thermal equilibrium, the ortho-to-para ratio of $\mathrm{D}_{2} \mathrm{O}$ is higher or equal to 2, depending on temperature (see Fig. 4 in Vastel et al. 2010). If the ortho-to-para $\mathrm{D}_{2} \mathrm{O}$ ratio estimated at $\sim 1.3$ in the absorbing layer really deviates from the $2: 1$ value as already suggested by Vastel et al. (2010), this could mean that some nuclear spin exchanges occur in the gas phase or at the grain surface. Indeed, the ortho-to-para ratio should be initially set during the molecule formation at a value reflecting the grain temperature $(\geq 2)$. Some spin exchange mechanisms would be consequently required to decrease the ortho-to-para ratio. These mechanisms are, however, poorly known. We can imagine some exchange mechanisms at the grain surface with interaction between electronic or nuclear spins. Unfortunately, the theoretical and laboratory measurements (Limbach et al. 2006; Pardanaud et al. 2007) are difficult to carry out. In the gas phase, no fast radiative transition is possible between ortho and para species, except via proton exchange with ions. For example, to modify the ortho-to-para ratio of water, collisions with $\mathrm{H}^{+}$and $\mathrm{H}_{3}^{+}$are required. For $\mathrm{D}_{2} \mathrm{O}$, the following reaction could consequently occur via $\mathrm{D}$ exchange between $\mathrm{D}_{2} \mathrm{O}$ and $\mathrm{H}_{2} \mathrm{D}^{+}$:

$\mathrm{o}-\mathrm{D}_{2} \mathrm{O}+\mathrm{H}_{2} \mathrm{D}^{+} \longleftrightarrow \mathrm{p}-\mathrm{D}_{2} \mathrm{O}+\mathrm{H}_{2} \mathrm{D}^{+}$.

However, this reaction could also lead to other products for which the branching ratios are unknown:

$\mathrm{D}_{2} \mathrm{O}+\mathrm{H}_{2} \mathrm{D}^{+} \longleftrightarrow \mathrm{HD}_{2} \mathrm{O}^{+}+\mathrm{HD}$

$\mathrm{D}_{2} \mathrm{O}+\mathrm{H}_{2} \mathrm{D}^{+} \longleftrightarrow \mathrm{D}_{3} \mathrm{O}^{+}+\mathrm{H}_{2}$. 
This reaction has never been studied experimentally or theoretically, but could be exothermic like the $\mathrm{H}_{2} \mathrm{O}+\mathrm{H}_{3}^{+}$reaction (Woon $\&$ Herbst 2009). The nuclear spin conversion could also be possible thanks to an exchange with $\mathrm{D}^{+}$.

\subsection{Water deuterium fractionation}

As already noticed in Coutens et al. (2012), the $\mathrm{HDO} / \mathrm{H}_{2} \mathrm{O}$ ratios determined in the hot corino and in the external absorbing layer are similar $(\sim 2-5 \%)$, although the corresponding densities are very different, a few $10^{8} \mathrm{~cm}^{-3}$ in the hot corino region and, typical of a molecular cloud, probably $10^{3}-10^{4} \mathrm{~cm}^{-3}$ in the external layer. Water shows a different behavior from other tracers as methanol $\left(\mathrm{CH}_{3} \mathrm{OH}\right)$ and formaldehyde $\left(\mathrm{H}_{2} \mathrm{CO}\right)$, whose deuteration increases with the density. Indeed, the deuteration of these heavier molecules that need CO ices to form is correlated with the $\mathrm{CO}$ depletion (linked to the molecular hydrogen density: Caselli et al. 1999; Bacmann et al. 2002) in prestellar cores (Bacmann et al. 2003, 2007). In contrast, the water fractionation ratio does not seem to vary with density. As discussed in Coutens et al. (2012), it is therefore possible that water was formed at low densities in the early stages of the star formation before the protostellar collapse.

To understand how the deuterium fractionation of water depends on the initial conditions of the cloud in which ices are forming, we used in the present study a grain surface chemical model (Cazaux et al. 2011), in which we considered the formation of ices by accreting species from the gas phase. The icy mantles then grow until $99 \%$ of $\mathrm{CO}$ and $\mathrm{O}$ from the gas are depleted onto dust. We set the initial densities of $\mathrm{O}$ and $\mathrm{CO}$ in the gas similar to densities of $\mathrm{O}$ and $\mathrm{CO}$ in translucent clouds, i.e., $n(\mathrm{O}) \sim n(\mathrm{CO}) \sim 1.5 \times 10^{-4} n_{\mathrm{H}}$ (Hollenbach et al. 2009). The gas phase species accrete on the dust and the chemical evolution of ices can be followed with time until most of $\mathrm{O}$ and $\mathrm{CO}$ are depleted from the gas phase $\left(t \sim 1-5 \times 10^{5}\right.$ years, depending on the density of the medium). During the formation of ices, $\mathrm{O}$ and $\mathrm{CO}$ gas phase abundances are decreasing while the abundances of gas phase $\mathrm{H}_{2}, \mathrm{H}$ and $\mathrm{D}$ are kept constant. The density of the cloud sets the gas phase $\mathrm{D} / \mathrm{H}$ ratio through ion-molecule chemistry (deuteration of $\mathrm{H}_{3}^{+}$sets the gas $\mathrm{D} / \mathrm{H}$ ratio, as described in Roberts et al. 2002). Therefore, a translucent cloud with an initial density of $10^{3}, 10^{4}$, and $5 \times 10^{4} \mathrm{~cm}^{-3}$ results in a $\mathrm{D} / \mathrm{H}$ ratio in the gas phase of $5 \times 10^{-3}, 5 \times 10^{-2}$, and $10^{-2}$ respectively (Cazaux et al. 2011). Consequently, we assume these three initial values for the density $\left(10^{3}, 10^{4}\right.$, and $\left.5 \times 10^{4} \mathrm{~cm}^{-3}\right)$ as well as three different temperatures $(12,15$, and $20 \mathrm{~K})$. The $\mathrm{HDO} / \mathrm{H}_{2} \mathrm{O}$ as well as the $\mathrm{D}_{2} \mathrm{O} / \mathrm{H}_{2} \mathrm{O}$ ratios obtained during the formation of ices are presented in Fig. 4. Both ratios are strongly sensitive to the dust temperature. Indeed, at low temperatures, $\mathrm{H}_{2}$ is much more abundant than $\mathrm{H}$ on the dust grains, and the successive hydrogenations of $\mathrm{O}$ with $\mathrm{H}_{2}$ dominate the formation of $\mathrm{H}_{2} \mathrm{O}$. The reaction $\mathrm{O}\left({ }^{3} \mathrm{P}\right)+\mathrm{H}_{2}$ is energetically not accessible at low temperatures since the reaction has a barrier of $0.57 \mathrm{eV}$ and is endo-ergic with an energy of $0.1 \mathrm{eV}$ for $\mathrm{H}_{2}(v=0$ ) (Weck et al. 2006). However, this reaction becomes exo-ergic for $v>0$ and the barrier decreases to $\sim 0.4 \mathrm{eV}$ for $v=1$ (Sultanov \& Balakrishnan 2005). The reaction $\mathrm{O}\left({ }^{3} \mathrm{P}\right)+\mathrm{H}_{2}(v=1)$ proceeds through tunneling at low temperatures, as shown by Weck et al. (2006). In our model, we consider only the reaction of $\mathrm{H}_{2}$ vibrationally excited with $\mathrm{O}\left({ }^{3} \mathrm{P}\right)$. For a population in equilibrium at a temperature $T_{\text {dust }}$, the fraction of molecules in the $J=1$ state is $\sim \exp \left(-150 / T_{\text {dust }}\right) \sim 3 \times 10^{-6}-5.5 \times 10^{-4}$ in the range of $T_{\text {dust }}$ considered in this study $(12-20 \mathrm{~K})$. The reaction through tunneling between $\mathrm{H}_{2}(v=1)$ and $\mathrm{O}\left({ }^{3} \mathrm{P}\right)$ dominates at very low temperatures when the dust is saturated with $\mathrm{H}_{2}$ molecules and the oxygen atoms present on the surface repetitively collide with $\mathrm{H}_{2}$. On the other hand, HDO forms through addition of atomic deuterium. These different formation routes imply that the $\mathrm{HDO} / \mathrm{H}_{2} \mathrm{O}$ ratio scales with $\mathrm{D} / \mathrm{H}_{2}$ ratio present in the gas, while the $\mathrm{D}_{2} \mathrm{O} / \mathrm{H}_{2} \mathrm{O}$ scales with $\left(\mathrm{D} / \mathrm{H}_{2}\right)^{2}$. The reaction between $\mathrm{O}$ and $\mathrm{H}$, however, dominates at $12 \mathrm{~K}$ at short timescale because of the relatively high initial $\mathrm{H} / \mathrm{H}_{2}$ ratio $\left(\sim 10^{-4}\right)$. However, the $\mathrm{H} / \mathrm{H}_{2}$ ratio decreases with time, since $\mathrm{H}$ atoms are quickly converted into $\mathrm{OH}$ and $\mathrm{H}_{2}$, and the reaction $\mathrm{O}+\mathrm{H}_{2}$ dominates (for $t \sim 10^{4}$ years at $n_{\mathrm{H}}=10^{3} \mathrm{~cm}^{-3}$ and for $t \sim 10^{5}$ years at $n_{\mathrm{H}}=10^{4} \mathrm{~cm}^{-3}$ ). If the grain is warm, the $\mathrm{H}_{2}$ molecules evaporate and the formation of $\mathrm{H}_{2} \mathrm{O}$ is insured by successive hydrogenation with $\mathrm{H}$ atoms. Since the formation of HDO involves atomic deuterium, the $\mathrm{HDO} / \mathrm{H}_{2} \mathrm{O}$ ratio becomes comparable to the $\mathrm{D} / \mathrm{H}$ ratio and the $\mathrm{D}_{2} \mathrm{O} / \mathrm{H}_{2} \mathrm{O}$ ratio comparable to $(\mathrm{D} / \mathrm{H})^{2}$. Therefore, the variation of the deuterium fractionation with the initial dust temperature is due to the different routes to form water molecules. In their modeling, Cazaux et al. (2011) found that the deuterium fractionation of the water ice mantle is not altered by the cloud collapse, pointing to the fact that the deuterium fractionation of water must have been set during the formation of ices.

The $\mathrm{HDO} / \mathrm{H}_{2} \mathrm{O}$ and $\mathrm{D}_{2} \mathrm{O} / \mathrm{H}_{2} \mathrm{O}$ observed ratios suggest that the ices present in IRAS 16293 and desorbing in the hot corino were made in a warm $(\sim 15-20 \mathrm{~K})$ and dense $\left(\sim 10^{4}-5 \times 10^{4} \mathrm{~cm}^{-3}\right)$ translucent cloud. Recently, Taquet et al. (2013) modeled the formation of deuterated water ice in cold conditions with a pseudo time-dependent multilayer approach (see Taquet et al. 2013, for a full description of the model). They showed that the relatively high deuterium fractionation of water could be attributed to the formation of water ices in dark phases (either at $n_{\mathrm{H}} \sim 10^{4} \mathrm{~cm}^{-3}$ at low temperatures, $\sim 10 \mathrm{~K}$, and high visual extinction, larger than $4 \mathrm{mag}$, or for higher densities, $\sim 10^{5} \mathrm{~cm}^{-3}$, at higher temperatures), where the $\mathrm{D} / \mathrm{H}$ ratio starts to efficiently increase. Although the chemical models diverge on the deuteration processes which are enhanced for warm dust in one case (Cazaux et al. 2011) and for cold dust in the other case (Taquet et al. 2013), our results agree that the high deuterium fractionation originates from a dense medium (both models need dense medium for high $\mathrm{D} / \mathrm{H}$ ratio).

In the extended absorbing layer, which is possibly due to photodesorption mechanisms, the observed $\mathrm{D}_{2} \mathrm{O} / \mathrm{H}_{2} \mathrm{O}$ ratio appears much larger compared to the hot corino value, in contrast to the $\mathrm{HDO} / \mathrm{H}_{2} \mathrm{O}$ ratio. It is also higher than all the chemical predictions. If the $\mathrm{D}_{2} \mathrm{O} / \mathrm{H}_{2} \mathrm{O}$ ratio observed in the gas phase of this layer really reflects the $\mathrm{D}_{2} \mathrm{O} / \mathrm{H}_{2} \mathrm{O}$ ratio at the grain surface, it would consequently suggest that water formed in a denser $\left(\gtrsim 5 \times 10^{4} \mathrm{~cm}^{-3}\right)$ region with warm $(\sim 20 \mathrm{~K})$ gas. A high density $\left(10^{5}-10^{6} \mathrm{~cm}^{-3}\right)$ is also required for the chemical modeling by Taquet et al. (2013). However, these results disagree with our observational constraints obtained from the density of this absorbing layer $\left(<10^{5} \mathrm{~cm}^{-3}\right.$, see Sect. 3). Nevertheless, we cannot exclude that, in this external layer, water can also be formed by gas phase mechanisms through ion-molecule reactions. This could also be one of the possible explanations of the difference between the $\mathrm{D}_{2} \mathrm{O} / \mathrm{H}_{2} \mathrm{O}$ ratios determined in the hot corino and in the absorbing layer. Other reasons can be mentioned to explain the discrepancy of the water deuterium fractionation between the hot corino and the absorbing layer. During the heating of the grain surfaces, an isotope exchange between water molecules may also occur at $150 \mathrm{~K}$ (Smith et al. 1997; Dulieu et al. 2010), leading to a decrease of the $\mathrm{D}_{2} \mathrm{O}$ abundance in the hot corino region. Also, in the hot corino, large columns of $\mathrm{H}_{2} \mathrm{O}, \mathrm{HDO}$, and $\mathrm{D}_{2} \mathrm{O}$ could self-shield these molecules. Since $\mathrm{H}_{2} \mathrm{O}$ is more 

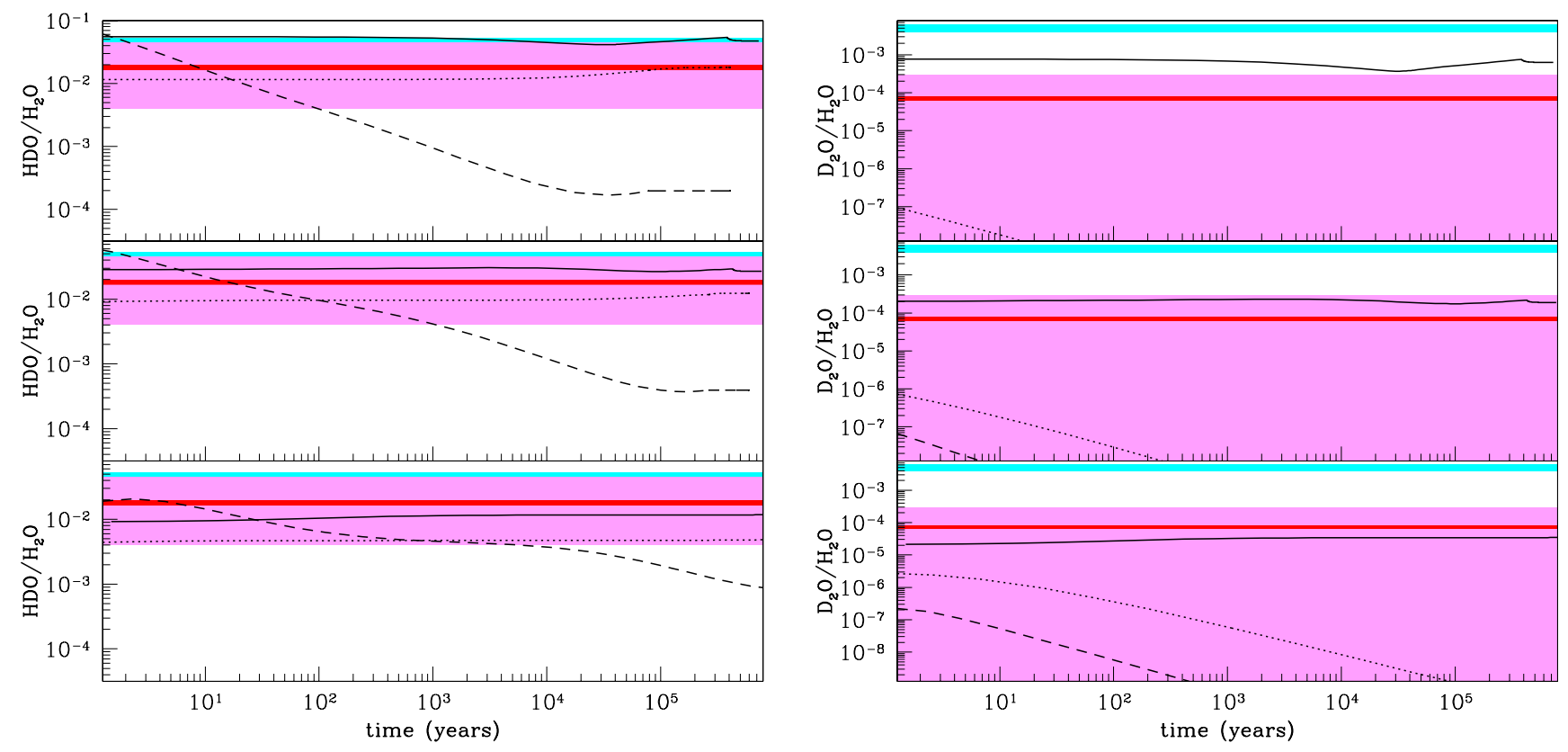

Fig. 4. Deuterium fractionation of water predicted by the grain surface chemical model (see text) assuming 3 initial dust temperatures $T_{\text {dust }}=12 \mathrm{~K}$ (dashed lines), $15 \mathrm{~K}$ (dotted lines) and $20 \mathrm{~K}$ (solid lines) and densities $n=10^{3}$ (lower panel), $10^{4}$ (middle panel) and $5 \times 10^{4} \mathrm{~cm}^{-3}$ (upper panel). The $\mathrm{HDO} / \mathrm{H}_{2} \mathrm{O}$ and $\mathrm{D}_{2} \mathrm{O} / \mathrm{H}_{2} \mathrm{O}$ ratios determined at $3 \sigma$ in the hot corino are indicated in pink with a red line for the best-fit value. The ratios of the absorbing layer are shown with a blue line.

abundant than its deuterated forms, it should self-shield first, reducing its photodissociation. Then if important columns of HDO and $\mathrm{D}_{2} \mathrm{O}$ can be reached, these molecules will be shielded. This implies a stratification of molecules and should favor the amount of water compared to its deuterated forms in environments where lots of water is present in the gas phase.

Recently, Persson et al. (2013) used interferometric (SMA and ALMA) observations of one HDO transition $(226 \mathrm{GHz})$ and two $\mathrm{H}_{2}{ }^{18} \mathrm{O}$ transitions $(203$ and $692 \mathrm{GHz}$ ) to determine the water deuterium fractionation in the hot corino of IRAS 16293. Water is only emitted by the core A and not by its binary component B. With their different method, Persson et al. (2013) derive a fractionation ratio about a factor of 10 lower than ours (Coutens et al. 2012). The origin of the divergence between these results is not clear, but could arise from the uncertain inner structure of the source. Indeed, a recent publication by Pineda et al. (2012) mentions that ALMA observations towards the source A would be consistent with the rotation of a disk, which is close to being edge-on. None of the two studies assume such a complex structure in their modeling, maybe implying the divergence between the estimated $\mathrm{HDO} / \mathrm{H}_{2} \mathrm{O}$ ratios. Compared with the predictions of the chemical model by Cazaux et al. (2011), the ratio determined by Persson et al. (2013) would require a low initial dust temperature (around $12 \mathrm{~K}$ ), in contrast to our results. But doubly deuterated water has never been detected with interferometers, making it not possible to check whether the $\mathrm{D}_{2} \mathrm{O} / \mathrm{H}_{2} \mathrm{O}$ ratio is also in agreement with an origin on cold dust grains and provide more constraints on the initial density. Consequently, complementary $\mathrm{D}_{2} \mathrm{O}$ observations at high spatial resolution would be necessary to assess precisely the conditions in which ices are forming with a method based on interferometric data as in Persson et al. (2013).

\section{Conclusions}

Thanks to the numerous HDO and $\mathrm{H}_{2}{ }^{18} \mathrm{O}$ transitions observed with the HIFI instrument on board the Herschel Observatory, we were able (Coutens et al. 2012) to constrain the abundances of these species from the hot corino throughout the envelope of the IRAS 16293 protostar to the external absorbing layer. In the present study, we have used the same non-LTE radiative transfer modeling to constrain the $\mathrm{D}_{2} \mathrm{O}$ abundances, employing the previous detections of the para and ortho fundamental transitions (Butner et al. 2007; Vastel et al. 2010) as well as the tentative detection of the higher energy para- $\mathrm{D}_{2} \mathrm{O} 2_{1,2}-1_{0,1}$ transition at $898 \mathrm{GHz}$ in Herschel/HIFI band 3b. To estimate the abundances, we used the full set of $\mathrm{D}_{2} \mathrm{O}$ collisional rate coefficients computed with ortho- and para- $\mathrm{H}_{2}$ (Faure et al. 2012), which were not available for the original detections. The results are presented in Table 2. The best-fit inner abundance $X_{\mathrm{in}}\left(\mathrm{D}_{2} \mathrm{O}\right)$ is about $7 \times 10^{-10}$, whereas the best-fit outer abundance $X_{\text {out }}\left(\mathrm{D}_{2} \mathrm{O}\right)$ is about $5 \times 10^{-12}$. The absorbing $\mathrm{D}_{2} \mathrm{O}$ components cannot be reproduced without taking into account the external absorbing layer. The column density in this layer is about $2.5 \times 10^{12} \mathrm{~cm}^{-2}$, leading to an abundance ranging from $6.6 \times 10^{-10}$ to $2.7 \times 10^{-9}$ if the lines are produced in a photodesorption layer at $A_{V} \sim$ 1-4 mag. We then compared the $\mathrm{HDO} / \mathrm{H}_{2} \mathrm{O}$ and $\mathrm{D}_{2} \mathrm{O} / \mathrm{H}_{2} \mathrm{O}$ observed ratios with the predictions of a grain surface chemical model (Cazaux et al. 2011), in which we considered the formation of ices by accreting species from the gas phase. Our estimations of the $\mathrm{HDO} / \mathrm{H}_{2} \mathrm{O}$ and $\mathrm{D}_{2} \mathrm{O} / \mathrm{H}_{2} \mathrm{O}$ ratios suggest that the ices present in IRAS 16293 and desorbing in the hot corino were made in a warm $(\sim 15-20 \mathrm{~K})$ and dense $\left(10^{4}-5 \times 10^{4} \mathrm{~cm}^{-3}\right)$ translucent cloud. The observed $\mathrm{D}_{2} \mathrm{O} / \mathrm{H}_{2} \mathrm{O}$ ratio appears much larger in the absorbing layer than in the hot corino, and we explored tentative explanations.

Acknowledgements. HIFI was designed and built by a consortium of institutes and university departments from across Europe, Canada, and the United States under the leadership of SRON Netherlands Institute for Space Research, Groningen, The Netherlands and with major contributions from Germany, France and the US. Consortium members are: Canada: CSA, U. Waterloo; France: IRAP (formerly CESR), LAB, LERMA, IRAM; Germany: KOSMA, MPIfR, MPS; Ireland, NUI Maynooth; Italy: ASI, IFSI-INAF, Osservatorio Astrofisico di Arcetri-INAF; Netherlands: SRON, TUD; Poland: CAMK, CBK; Spain: Observatorio Astronómico Nacional (IGN), Centro de Astrobiología 
(CSIC-INTA). Sweden: Chalmers University of Technology - MC2, RSS \& GARD; Onsala Space Observatory; Swedish National Space Board, Stockholm University - Stockholm Observatory; Switzerland: ETH Zurich, FHNW; USA: Caltech, JPL, NHSC. We thank the CNES (Centre National d'Études Spatiales) for its financial support. V.W. thanks the French National Program PCMI for the partial funding of her research.

\section{References}

Bacmann, A., Lefloch, B., Ceccarelli, C., et al. 2002, A\&A, 389, L6 Bacmann, A., Lefloch, B., Ceccarelli, C., et al. 2003, ApJ, 585, L55

Bacmann, A., Lefloch, B., Parise, B., Ceccarelli, C., \& Steinacker, J. 2007, in Molecules in Space and Laboratory

Bacmann, A., Caux, E., Hily-Blant, P., et al. 2010, A\&A, 521, L42

Bottinelli, S., Ceccarelli, C., Neri, R., et al. 2004, ApJ, 617, L69

Brünken, S., Müller, H. S. P., Endres, C., et al. 2007, Phys. Chem. Chem. Phys., 9, 2103

Butner, H. M., Charnley, S. B., Ceccarelli, C., et al. 2007, ApJ, 659, L137

Caselli, P., Walmsley, C. M., Tafalla, M., Dore, L., \& Myers, P. C. 1999, ApJ, 523, L165

Caux, E., Kahane, C., Castets, A., et al. 2011, A\&A, 532, A23

Cazaux, S., Caselli, P., \& Spaans, M. 2011, ApJ, 741, L34

Ceccarelli, C., Castets, A., Caux, E., et al. 2000, A\&A, 355, 1129

Ceccarelli, C., Bacmann, A., Boogert, A., et al. 2010, A\&A, 521, L22

Coutens, A., Vastel, C., Caux, E., et al. 2012, A\&A, 539, A132

Crimier, N., Ceccarelli, C., Maret, S., et al. 2010, A\&A, 519, A65

Daniel, F., Dubernet, M.-L., \& Grosjean, A. 2011, A\&A, 536, A76

de Graauw, T., Helmich, F. P., Phillips, T. G., et al. 2010, A\&A, 518, L6

Dulieu, F., Amiaud, L., Congiu, E., et al. 2010, A\&A, 512, A30

Faure, A., Wiesenfeld, L., Scribano, Y., \& Ceccarelli, C. 2012, MNRAS, 420, 699
Fraser, H. J., Collings, M. P., McCoustra, M. R. S., \& Williams, D. A. 2001, MNRAS, 327, 1165

Frerking, M. A., Langer, W. D., \& Wilson, R. W. 1982, ApJ, 262, 590

Hily-Blant, P., Maret, S., Bacmann, A., et al. 2010, A\&A, 521, L52

Hogerheijde, M. R., \& van der Tak, F. F. S. 2000, A\&A, 362, 697

Hollenbach, D., Kaufman, M. J., Bergin, E. A., \& Melnick, G. J. 2009, ApJ, 690 1497

Limbach, H.-H., Buntkowsky, G., Matthes, J., et al. 2006, Chem. Phys. Chem., 7,551

Linsky, J. L., Draine, B. T., Moos, H. W., et al. 2006, ApJ, 647, 1106

Müller, H. S. P., Schlöder, F., Stutzki, J., \& Winnewisser, G. 2005, J. Mol. Struct., 742,215

Ott, S. 2010, in Astronomical Data Analysis Software and Systems XIX, eds. Y. Mizumoto, K.-I. Morita, \& M. Ohishi, ASP Conf. Ser., 434, 139

Pardanaud, C., Michaut, X., Fillion, J.-H., Vasserot, A.-M., \& Abouaf-Marguin, L. 2007, in Molecules in Space and Laboratory

Parise, B., Du, F., Liu, F.-C., et al. 2012, A\&A, 542, L5

Persson, M. V., Jørgensen, J. K., \& van Dishoeck, E. F. 2013, A\&A, 549, L3

Pilbratt, G. L., Riedinger, J. R., Passvogel, T., et al. 2010, A\&A, 518, L1

Pineda, J. E., Maury, A. J., Fuller, G. A., et al. 2012, A\&A, 544, L7

Roberts, H., Herbst, E., \& Millar, T. J. 2002, MNRAS, 336, 283

Roelfsema, P. R., Helmich, F. P., Teyssier, D., et al. 2012, A\&A, 537, A17

Scribano, Y., Faure, A., \& Wiesenfeld, L. 2010, J. Chem. Phys., 133, 1105

Smith, S. R., Huang, C., \& Kay, B. D. 1997, J. Phys. Chem. B, 101, 6123

Sultanov, R. A., \& Balakrishnan, N. 2005, ApJ, 629, 305

Taquet, V., Peters, P. S., Kahane, C., et al. 2013, A\&A, 550, A127

Vastel, C., Ceccarelli, C., Caux, E., et al. 2010, A\&A, 521, L31

Weck, P. F., Balakrishnan, N., Brandão, J., Rosa, C., \& Wang, W. 2006, J. Chem. Phys., 124, 4308

Woon, D. E., \& Herbst, E. 2009, ApJS, 185, 273 